\title{
Peptide Phage Display: Molecular Principles and Biomedical Applications
}

Therapeutic Innovation \& Regulatory Science 2020, Vol. 54(2) 308-317 (C) The Author(s) 2020 https://doi.org//0.1007/s4344I-019-00059-5

\author{
Marlon S. Zambrano-Mila', Karen Elizabeth Sánchez Blacio', \\ and Nelson Santiago Vispo' ${ }^{\circledR}$
}

\begin{abstract}
Phage display (PD) is a technology based on the presentation of functional exogenous peptides on the capsid surface of bacteriophages. PD is performed by introducing a DNA sequence of interest at a specific position within a functional viral gene. In addition, peptide phage libraries are powerful tools for expressing a wide range of random peptides and for specific peptide screening. Specifically, PD applications include the analysis of binding and interactions between proteins, the identification of bioactive peptides that bind to receptors, the identification of disease-associated antigens, and the identification of cell-specific peptides. Since its emergence, PD technology has revolutionized several fields in the biological sciences, such as oncology, cell biology, and pharmacology, the innumerable applications for which will be described throughout this review.
\end{abstract}

\section{Keywords}

phage display, peptide libraries, cancer, infection diseases, drug delivery system, clinical trials

\section{Introduction}

Phage display (PD) technology was invented by George Smith in 1985 (Chemistry Nobel Prize 2018). Since its introduction, this technique has revolutionized several biological fields because of the use of its relatively fast evolution as powerful mechanism for the production of large numbers of proteins, ${ }^{1}$ the isolation of functional and biological compounds, the analysis of protein-protein interactions, ${ }^{2}$ and the study of antigenantibody binding. ${ }^{3}$ The aim of this review is to provide an overall framework regarding the current applications of PD in some selected fields, such as oncology, cell biology, drug discovery, and delivery systems.

Filamentous Bacteriophages: Infectious Cycle and Phage Display Technology

Phage display (PD) technology exploits the nature and the infectious cycle of filamentous bacteriophages (viruses that infect bacteria) such as M13 (see Figure 1). The M13 bacteriophage is the most frequently used filamentous phage in PD applications and is characterized by having a cylindrical capsule that is primarily made up of the protein pVIII. The ends of the capsid contain copies of different proteins, such as 5 copies of the pIII and pVI proteins at one end and 3 to 5 copies of the $\mathrm{pVII}$ and pIX proteins at the opposite end. The viral infection cycle begins with the attachment of the pIII protein to the bacterium and the subsequent injection of the single-stranded DNA bacteriophage genome into the bacterium. Once inside the bacterium, the single-stranded DNA is converted into double-stranded DNA (the replicative form of DNA) by the replication machinery of the host organism. Subsequently, the DNA is replicated by rolling circle replication, and the resulting product is a single-stranded DNA molecule encoding the proteins necessary for DNA packaging into the bacteriophage capsid. ${ }^{4}$ Finally, the mature bacteriophages exit the bacterium without lysing the cell, which is a characteristic feature of filamentous phages.

Phage display is an efficient molecular technique in which the desirable peptides that are fused to the viral coat proteins are displayed on the surface of a bacteriophage, such as the M13 phage. In the process of $\mathrm{PD}$, the foreign peptides are generally fused to the N-terminal end of the pIII and pVIII proteins $^{5,6}$ (see Figure 1). The foreign peptide fragments range from 6 to 43 amino acids in length, since peptides that are too large can potentially interfere with the infectious activity of the virus or with capsid assembly. ${ }^{2}$

\footnotetext{
'School of Biological Sciences and Engineering, Yachay Tech University, San Miguel de Urcuquí, Ecuador
}

Submitted 29-Sep-2018; accepted I5-Feb-2019; published online 6-Jan-2020

\section{Corresponding Author:}

Nelson Santiago Vispo, School of Biological Sciences and Engineering, Yachay Tech University, San Miguel de Urcuquí, Imbabura 100I50, Ecuador.

Email: nvispo@yachaytech.edu.ec 


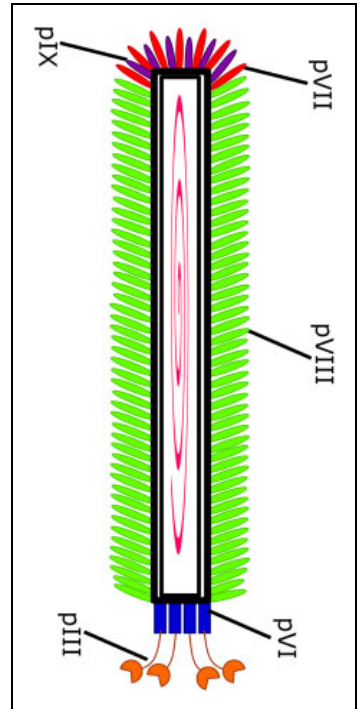

Figure I. Schematic of a filamentous phage displaying exogenous peptide. The MI 3 capsid, which encapsulates the ssDNA genome, consists of 2700 copies of pVII. This major protein is commonly used to display exogenous peptides or proteins on the bacteriophage surface. Approximately 5 copies of the coat proteins PVII and pIX cap one end of phage while the other end consists of 3 or 5 copies of plll and $\mathrm{pVI}$ proteins. (ssDNA, single-stranded DNA.)

\section{PD Technology Is the Result of 2 Approaches}

An important aspect of PD technology is the introduction of a gain-of-function mutations (the insertion of a sequence of interest) at specific position within a functional viral gene that retain the functionality of its protein product and results in modified peptides displayed on the viral surface. For random nucleotide insertions, the resulting viral particles form a phage-displayed peptide library that may contain up to $10^{10}$ different peptides constructed simultaneously. The generation of a library of peptides is based on the triplet rule as well as the degenerative nature of the genetic code, where the alteration of amino acid sequences can result in a large and diverse repertoire of random peptides fused to capsid proteins. ${ }^{7}$ Thus, this large variability makes it possible to construct libraries of peptides, proteins, antibody fragments, or enzymes.

Subsequently, these libraries undergo a screening procedure in which binding clones are selected from nonbinding clones through affinity purification. ${ }^{8}$ Peptides that bind to target molecules can be identified by biopanning (a type of affinity selection) (see Figure 2). ${ }^{7}$ It is worth mentioning that the development of a PD peptide library is a key step in successful screening, because the probability of selecting a ligand that binds to a target molecule is related to the PD library diversity and the size of the insert. 9

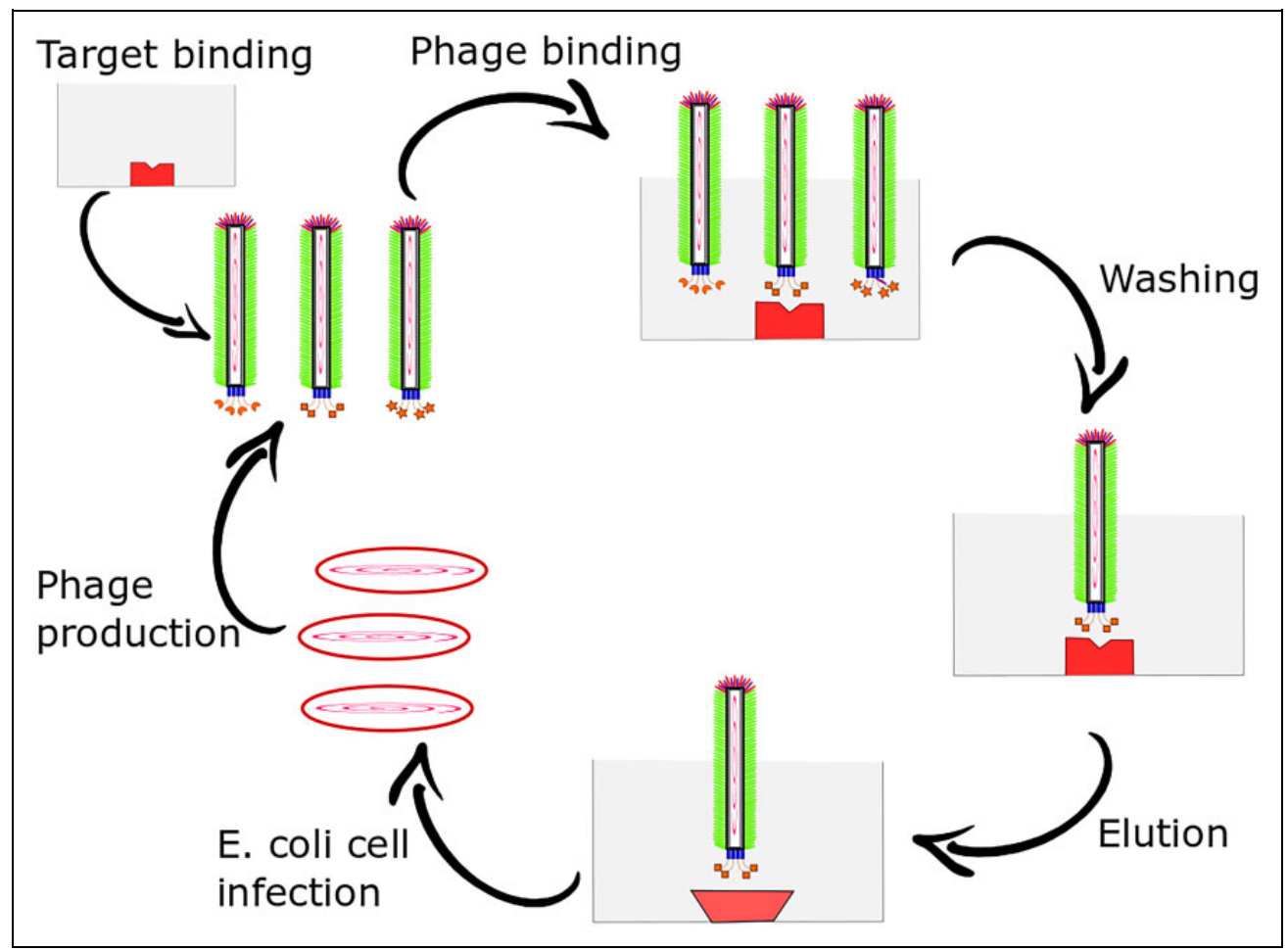

Figure 2. Biopanning: An affinity selection procedure used to filter out target phage-displayed peptides. During the biopanning process, PD libraries are incubated with immobile target antigens on a solid plate. The phage particles are linked to the antigens, while the phages that did not bind are washed away. The bound phage particles are subsequently eluted and amplified by lysogenic infection of bacteria. (PD, phage display.) 
These aspects of PD technology make it a highly useful biotechnological technique, allowing for analyses of the binding and interactions between proteins, the identification of bioactive peptides that bind to receptors, the identification of disease-associated antigens, and the identification of cellspecific peptides as well as other applications in various medical fields.

\section{Medical Applications}

\section{Cancer and Metastatic Lesions}

In 2018, 9.6 million deaths were attributed to cancer, making it the second leading cause of death worldwide. ${ }^{10}$ Currently, one of the major challenges in cancer therapy has been the lack of an efficient means of targeting therapeutic drugs to tumor sites as well as a lack of therapeutic drugs with high specificity. An efficient mean facilitating the direct application of drugs would allow the target tumor cells to be eradicated while preventing healthy cells from being damaged. In this context, diverse cell membrane proteins, such as growth factor receptors, adhesion proteins, integrins, and other markers are potential targets for tumor-specific peptides and subsequent peptide therapy. ${ }^{11}$ Therefore, PD libraries can be large reservoirs of tumorspecific peptides capable of binding to tumor vasculature and cancer cells.

Breast cancer is the most frequent type of cancer in women in both developed and developing countries, with approximately 2,088,849 novel cases diagnosed in $2018 .{ }^{12}$ In breast cancer cells, acidic fibroblast growth factor (aFGF) is highly expressed, and its interactions with its receptors (FGFRs) promote the progression of the disease. An aFGF-binding peptide called AP8 was shown to interact with FGFRs as an antagonist and inhibited cell proliferation, as both breast cancer and vascular endothelial cells were observed to be arrested in the G0/G1 stage. ${ }^{13}$ Another cell-surface protein associated with the progression and metastasis of breast cancer cells is CD44. Novel peptides that had been screened from a peptide library were shown to bind to CD44 with high affinity. ${ }^{14}$ In addition, the expression of CD133, also known as Prominin 1, has been shown to be associated with cancer stem cells in humans and mice, making it a potential cancer biomarker. The peptide LS-7 (LQNAPRS) was screened and identified as a specific CD133binding ligand in a murine model, where in vivo experiments demonstrated its high specificity and affinity to murine CD133. Furthermore, LS-7 highly suppressed the migration of cancerous cells in colon and breast cancer. ${ }^{15}$ In addition, phage probes against breast cancer cells (MCF-7 and ZR-75 -1) ${ }^{16}$ and a novel peptide GYSASRSTIPGK, ${ }^{17}$ which were identified using 8- and 9-mer landscape phage libraries, were able to bind to breast cancer stem cells with high specificity. In some malignancies, such as breast cancer, the abnormal expression of epidermal growth factor receptor (Her2) has been observed. By biopanning phage display libraries against Her2, a spectrum of ligands exhibiting a variety of sequences and motifs were identified $^{18}$ that are potential starting points for generating highly specific Her2-binding peptides. Thus, PD libraries are diverse reservoirs of tumor-binding peptides that may be useful in developing new diagnostic approaches and potential therapeutic drugs.

In 2018, more than 1.2 million people were diagnosed with prostate cancer, a disease that is considered to be the most common male malignancy and is the second greatest cause of male cancer death in developed countries. ${ }^{12,19}$ Although a vast number of therapeutic drugs have been developed against prostate cancer, the efficacy of these drugs is still inappropriate in several cases. ${ }^{20}$ The use of landscape phage libraries resulted in the identification of 3 phage probes that bind to PC3 prostate cancer cells. The 3 phages, carrying the peptides DTDSHVNL, DTPYDLTG, and DVVYALSDD, showed high specificity and selectivity toward PC3 cells. ${ }^{21}$ In addition, a splice variant of CD44 is considered to be a potential biomarker for prostate cancer because of its role during cell adhesion and tumor progression. In this context, 4 novel peptides were identified that showed promising characteristics, including high specificity and superior binding to the CD44v6 target on prostate cancer cells. ${ }^{22}$ Fibroblast growth factor $8 \mathrm{~b}$ (FGF8b) is a specific isoform whose expression is related to tumor growth, angiogenesis, and the stage of prostate cancer. Twelve FGF8b-binding phage particles were identified by screening a PD library against FGF8b, and a peptide called P12 in particular was identified that may act as a growth-factor antagonist. ${ }^{23}$ The discovery of these target peptides may be useful for imaging and therapeutic applications, and phage probes may even have potential in either therapeutic treatments or the diagnosis of prostate cancer.

Ovarian cancer is characterized by its asymptomatic development and the fast propagation of metastatic cells, with $70 \%$ of patients diagnosed at late stages because of the current lack of effective biomarkers for the diagnosis of this gynecologic malignancy. ${ }^{24}$ Using a PD peptide library, novel peptides (such as WSGPGVWGASVK and NPMIRRQ) have been discovered that target ovarian cancer cells. ${ }^{25,26}$ In vitro experiments using the peptide WSGPGVWGASVK showed it to have excellent potential to be incorporated into both tumor cells and angiogenic endothelial cells. ${ }^{25,26}$ In addition, a tumor cell-binding peptide (SWQIGGN) was isolated in ovarian cancer cells and was assessed for its cell adhesion, spreading, motility, and invasion characteristics. The results of in vitro experiments showed that this peptide was able to inhibit cancer cell invasion, proliferation, adhesion, and migration. ${ }^{27}$ Furthermore, the results of in vivo assays demonstrated that the SWQIGGN peptide played an inhibitory role toward neoplasm growth and metastasis. ${ }^{27}$ In another study, an integrated microfluidic system was developed to facilitate the screening of a PD library for cell-specific peptides showing high affinity and specificity toward ovarian cancer cells. ${ }^{28}$ These remarkable findings may be used for the development of novel diagnostic and treatment strategies.

In 2018, a total of 2,093,876 human deaths were attributed to lung cancer and 1,761,007 new cases were diagnosed 
worldwide. ${ }^{12}$ The conventional diagnosis lung cancer through lung tumor-specific biomarkers is ineffective and expensive. To address this issue, human lung diagnostic protein chips with novel screened biomarkers were developed using a T7-phage cDNA library, allowing patients with lung cancer to be diagnosed at the early stages of this disease. ${ }^{29}$ Through in vivo screening of peptide-displaying phages, novel peptides targeting human lung cancer have been discovered using a mouse model to mimic the lung tumor environment. ${ }^{30,31}$

PD technology has also been an important tool in the identification of novel peptides targeting other types of cancerous cells. One of the most frequent malignancies that develops along the genitourinary tract is bladder cancer. In one study, the peptide CSNRDARRC was shown to be able to specifically bind to cultured bladder tumor cells. ${ }^{32}$ In another study, the peptide OSP-1 was screened from a PD library and shown to specifically bind to osteosarcoma cells, with a target binding site that may be related to heparan sulfate proteoglycans. Therefore, a properly labeled OSP-1 peptide may have excellent potential to act as efficient probe for cancer imaging. ${ }^{33}$ Using a landscape phage display library, phages displaying proteins with specificity toward cell membrane markers of glioma cells in rats were identified. These results may allow glioma tissues to be profiled so that the therapeutic effectiveness of drugs can be increased and their toxicity decreased by designing anticancer compounds based on tumor tissue profiles. ${ }^{34}$ Furthermore, following hepatitis infection, chronically infected livers may develop hepatocellular carcinoma. In this context, the use of a random phage display peptide library allowed potential biomarkers to be identified, such as an $\mathrm{HC} 1$ mimic peptide for early hepatocellular carcinoma. ${ }^{35} \mathrm{~A}$ novel peptide called AAD (AADNAKTKSFPV) that exhibits high binding specificity toward gastric cancer cells was identified by biopanning the $\mathrm{PhD}-12$ phage library. This peptide has the potential to be used to differentiate neoplastic and normal gastric mucosa, leading to an effective approach for cancer diagnosis via endoscopy at early stages. ${ }^{36}$ Additionally, PD provides a high-throughput means of screening phage fusion proteins that are selective and specific toward PANC-1 pancreatic tumoral cells. ${ }^{37}$ In summary, the peptides described above may act as a moiety for the targeted delivery of drug therapeutics or for the diagnosis or imaging of different types of cancer because of their highly specific targeting capabilities.

\section{Parasitic Infectious Diseases}

Infectious agents have become an important cause of illness and death, accounting for 15 million deaths worldwide in 2010 according to the World Health Organization. ${ }^{38}$ Paracoccidioidomycosis (PCM) is an endemic systemic disease in Latin America caused by the fungus Paracoccidioides brasiliensis. PD screening is a useful tool for identifying epitopes that may be used for the serodiagnosis of PCM. ${ }^{39}$ In addition, leprosy is a chronic infectious disease caused by Mycobacterium leprae for which early detection is difficult because of the lack of a laboratory test. A number of peptide ligands were identified from leprosy patients, from which a set of 3 peptides allowed for patients to be successfully diagnosed with multibacillary leprosy. ${ }^{40}$ Thus, these peptides may have potential use in the design of leprosy diagnostic serologic assays.

Leishmania parasites are the etiological agents of different visceral and cutaneous diseases located in tropical and subtropical countries. Using random peptide PD libraries, 3 peptides were identified as candidate antigens for Leishmania braziliensis. These peptides presented high performance and sensitivity, either alone or in combination, indicating that they may have applications in the immunoprotection against or diagnosis of leishmaniasis. ${ }^{41}$ Canis lupus familiaris is an important reservoir of Leishmania infantum, and a novel peptide was identified that showed a promising capability to promote a state of active immunity against $L$ infantum infections in mice after immunized using liposomes as a vaccine vehicle, ${ }^{42}$ The abovementioned findings reveal a promising health care target with respect to the serodiagnosis and development of vaccines against Leishmania parasites.

Worldwide, 212 million new cases of malaria were diagnosed and approximately 429000 people died from this infectious disease in $2015 .{ }^{43}$ Plasmodium falciparum and Plasmodium knowlesi are the causative parasites of malaria, a life-threatening disease in humans. These parasites infect red blood cells during the infectious process, causing physical and chemical changes in the plasma membrane of erythrocytes. A phage display library was screened on the surface of infected red blood cells, resulting in the identification of a peptide (LVDAAAL) that is a target compound for the development of antimalarial agents. ${ }^{44}$ With respect to Plasmodium knowlesi, merozoite surface protein-142 has been a target for the development of a vaccine and for the diagnosis of malaria. In this context, a synthetic peptide library and a phage display library were used to identify and map relevant epitopes. This approach resulted in the identification of 2 epitopes (TAKDGMEYYNKMGELYKQ and RCLLGFKEVGGKCVPASI) that may be potential candidates for immunodiagnostic assays and vaccine design. ${ }^{45}$ Finally, a new interaction between the MSP1 N-terminus and glycophorin A of red blood cells was reported, showing that the MSP1-glycophorin A complex is essential during the adhesion stage of the parasitic invasion of red blood cells. ${ }^{46}$

The process by which the malaria parasite is fertilized in the mosquito midgut and the corresponding molecular events has been poorly studied and understood. However, female gamete peptide 1 was shown to bind to the surface of Plasmodium berghei gametes and suppress oocyst formation, making it a potentially useful molecule in the development of innovative transmission-preventing strategies. ${ }^{47} \mathrm{~A}$ novel peptide was identified called peptide-salivary gland and midgut peptide 1 (SM1) that specifically binds to the lobes of salivary glands and the midgut epithelium of mosquito. Furthermore, it was shown that parasitic invasion of salivary glands was inhibited by the peptide SM1. ${ }^{48}$ The components of the molecular 
apparatus involved in the interaction between host cells and the parasite Plasmodium during its life cycle are common targets and candidates for drug and vaccine development. Using PD technology, the insert EWGWS was identified in Plasmodium knowlesi ookinete surface enolase and was shown to bind to a conserved motif (PWWP) present in ookinete-binding peptides. ${ }^{49}$ The findings may be useful for genetically manipulating the ability of the host mosquito to act as a vector of the parasite Plasmodium knowlesi.

PD technology has been a useful biotechnological tool in the identification and characterization of peptides involved in the infectious process in human and in different animals. For instance, severe intestinal disorders in young goats can be caused by infections by the parasite Eimeria ninakohlyakimovae. A new method used to identify peptides binding to the surface of caprine umbilical vein endothelial cells was described and provided evidence that caprine endothelial cell peptide 2 (PCEC2) and PCEC5 may reduce the E ninakohlyakimovae infection rate by hindering sporozoite invasion. ${ }^{50}$ These findings may contribute to the production of anticoccidial drug or the development of novel vaccine development strategies.

\section{Viral Infectious Diseases}

A remarkable aspect of the potential of PD libraries lies in the ability to use them to map epitopes of some types of viruses and even the possibility of using screened epitopes as a subunit vaccine. For example, rabies virus glycoprotein and nucleoprotein are essential for increasing the protective immunity of the host. Thus, anti-rabies viral IgG from immunized dogs was used to screen target peptides that mimic glycoprotein and nucleoprotein epitopes. The results showed that the RYDDW-T motif may act as a new epitope region. ${ }^{51}$ In addition, a novel approach for the detection of hepatitis A virus based on phage-displayed peptides was developed and allowed ligands that mimic viral antigens to be identified. ${ }^{52}$ Nipah virus (NiV) is a pathogenic zoonotic paramyxovirus whose infection cycle initiates after the binding of $\mathrm{G}$ glycoprotein to the host receptor. The NiV G glycoprotein cell-binding domain was mapped using a PD system, and the results showed that NiV G amino acids 498-602 play an essential role in binding to the host. ${ }^{53}$ These discoveries are essential for the development of a human-targeting vaccine or diagnostic compounds for the aforementioned viral diseases.

From the standpoint of the host animals, target peptides involved in viral infectious events have been identified and characterized with the help of PD. For instance, porcine reproductive and respiratory syndrome is a widespread disease affecting domestic pigs and whose etiologic agent is porcine reproductive and respiratory syndrome virus (PPRSV). Using PD screening technology against PPRSV polymerase, a novel peptide (SPHIIRNHRLSK) was identified, ${ }^{54}$ and subsequent antiviral and cytotoxic activity assays revealed it to have high antiviral activity through its ability to bind to the PRRSV polymerase. Moreover, this peptide showed a relatively low toxicity toward cells in which viral replication was inhibited. ${ }^{54}$ Furthermore, classical swine fever (known as pig plague) caused by classical swine fever virus is a highly contagious disease affecting both domestic and wild swine. Phage ligands against the CSFV E2 protein were screened from an $\mathrm{f} / 8$ landscape PD library, and 4 E2-specific clones displaying the sequence DRATSSNA were able to inhibit CSFV replication in the cell line PK-15. ${ }^{55}$ These important discoveries may be the bases for the development of vaccines, antiviral drugs, and epidemiologic strategies to avoid the devastating economic consequences of these infections in the swine industry worldwide.

\section{Degenerative Joint Disorders}

Phage display random peptides library has allowed serum biomarkers to be identified for ankylosing spondylitis, which is a chronic rheumatic disorder characterized by inflammation of the joints of the spine. ${ }^{56}$ In addition, a phage-displayed random peptide library allowed serum biomarkers such as peptide KOA1 to be screened from patients with knee osteoarthritis, which is characterized by the continuous degeneration of joint cartilage and new bone development. ${ }^{57}$ Rheumatoid arthritis is a chronic inflammatory autoimmune disease that does not currently have a proper and definitive test for its diagnosis. To address this issue, an M12-displayed peptide capable of binding to the protein carbonic anhydrase III was identified with excellent specificity and sensitivity that may be useful as an antigen for the diagnosis of rheumatoid arthritis. ${ }^{58}$ Transforming growth factor $\beta 1$ has a wide range of functions, including the control of cell growth, proliferation, differentiation, and apoptosis, and it is especially involved in bone formation, promoting stem cell differentiation and osteogenesis. Novel peptides of TGF- $\beta 1$ (T $\beta \mathrm{ms}$ ) were identified from a PD library that allow for the modification of TGF- $\beta 1$ signal transduction. The screened peptides encourage early osteoblast proliferation and decrease the inhibitory activity of TGF- $\beta 1$ toward osteogenic differentiation in the late stages of degenerative joint disorders. ${ }^{59}$ Furthermore, T $\beta \mathrm{ms}$ could be used on implant faces to improve bone remodeling.

\section{Cardiac Diseases}

In acute myocardial infarction and other heart diseases, troponin I is considered to be a biomarker for myocardial injury because of its high specificity and sensitivity. PD was used to identify unique peptide motifs that recognize both the human and rat forms of troponin I. These peptides may be essential for future clinical assays used in the diagnosis of heart injuries as well as in controlling cardiac cell growth in culture. ${ }^{60}$ Heart failure can be associated with the inappropriate activation of mineralocorticoid receptors in the heart. Mineralocorticoid receptor-interacting proteins were screened for using a T7 PD library, and eukaryotic elongation factor 1A1, x-ray repair cross-complementing protein 6 , and structure-specific recognition protein 1 were identified as novel 
mineralocorticoid receptor coactivators. ${ }^{61}$ In addition, biopanning a PD peptide library against myoglobin allowed for the identification of markers that could be used in the early assessment of acute myocardial infarction, such as the peptides 3R7 (CPSTLGASC), 3R1 (CNLSSSWIC), and 3R10 (CVPRLSAPC). ${ }^{62}$

\section{Brain Injuries}

The currently available diagnostic approaches are inefficient at detecting the presence and extent of traumatic brain injuries. PD technology was used to identify serum-associated traumatic brain biomarkers, such as glial fibrillary acidic protein, allowing the severity of brain injury damage to be detected and for recovery and therapeutic efficiency predictions to be made. ${ }^{63}$ Additionally, PepC7 may act as highly selective peptide ligand that is specific for the brain vascular receptors in in vivo analysis and could be adapted for use in a drug delivery system in the brain. ${ }^{64}$

A great deal of research in other medical and health fields have utilized PD technology. For instance, endometriosis, a benign disease characterized by external outgrowth of the uterus-lining tissue (endometrium) in ovaries, fallopian tubes, intestines, and bladder, affects women during their reproductive life. Novel peptides, especially RTRLHTR, which specifically bound to ectopic endometrium were isolated from a 7-mer phage display library, opening up new directions for the early diagnosis of endometriosis and the development of proper medical treatments for this disease. ${ }^{65}$ In addition, because the regeneration of skin from severe injuries is currently a great challenge because of the limitations of the available treatment methods, the development of novel skin regeneration therapeutics is a currently active area of research. A novel small peptide called H1 that targets fibroblast growth factor receptor 2 IIIc was identified as a promising candidate for skin wound healing because of its high binding affinity. Additionally, H1 improved both the proliferation and cellular motility of fibroblasts and vascular endothelial cells in vitro and promoted skin wound healing in a murine wound model in vivo. ${ }^{66}$ Based on these attributes, the peptide H1 may be a good therapeutic drug candidate for repairing skin wounds. Although insulin plays a major role in blood sugar control, it is involved in many other physiological events, such as the proliferation, differentiation, and migration of fibroblasts and keratinocytes, which are essential for skin health and wound healing. Therefore, a current challenge is the identification of therapeutic compounds that inhibit insulin-degrading enzyme (IDE). In this context, PD was used to identify new peptide inhibitors of this endopeptidase, one of which greatly inhibited insulin degradation and enhanced insulin-associated events, such as the transcription, translation, and secretion of collagen in mice fibroblasts as well as keratinocyte migration, demonstrating the great potential of this peptide in therapeutic and cosmetic applications as a topical drug. ${ }^{67}$ Activin receptor type $2 \mathrm{~B}$, commonly known as ActRIIB, is an activin type 2 receptor involved in cell metabolism proliferation, and differentiation, and whose protein interactions are considered targets for the medical treatment of muscular dystrophy and sarcopenia. Peptides with ligandselective ActRIIB antagonist activity were screened from a PD library and were shown to bind to the extracellular domain of ActRIIB, ${ }^{68}$ inhibiting protein-protein interactions between ActRIIB and its ligands.

\section{Applications of Phages as Drug Delivery Systems}

Currently, pharmaceutical technology allows peptides and proteins to be delivered to target cell, tissues, or organs via the parenteral, buccal, transdermal, rectal, vaginal, and nasal pathways. ${ }^{3}$ However, the delivery of drugs to target organs or tissues is constrained by physical and physiological barriers, limitations that directly reduce the therapeutic index of drugs and even cause the rise of drug resistance in the case of infectious diseases. Therefore, spatiotemporally controlled delivery systems are needed that are needed to improve the pharmacokinetic and pharmacodynamic features of drugs. To fill this need, viral particles can be used as delivery vehicles, such as the M13 phage, which has been used to combat bacterial diseases through the transport of DNA. This DNA sequence encodes Gef, a 50-amino acid cell-membrane protein that enables cell respiration arrest and for the apoptosis to be triggered, and ChpBK, which plays a role as an mRNA interferase by cleaving RNA, and its use resulted in the lysis of target bacteria in in vitro assays and in a mouse model. ${ }^{69}$ Drugcarrying phages are an innovative type of nanomedicine that couple biologic and chemical constituents into a drug delivery system. Antibacterial phages have been used to successfully carry antibacterial agents such as the antibiotic chloramphenicol. These viral particles are nontoxic and had a greatly reduced immunogenicity in a mouse model. ${ }^{70}$ Furthermore, the complete growth inhibition of the bacteria Staphylococcus aureus, Streptococcus pyogenes, and Escherichia coli was demonstrated using filamentous phages as target drug carriers. $^{71}$ Additionally, 2 novel bacteriophages (Str01 and Str03) that belong to the Siphoviridae family showed activity against health-threatening group A Streptococcus. ${ }^{72}$

Important features, such as increased pharmacokinetics, stable formulation, and "passive" targeting of tumor cells are characteristics of liposomal drug delivery systems. Peptideassociated liposomes consist of 3 major parts: a drug peptide, a liposome carrier, and specific ligands. A novel feature of liposomes as nanocarriers was designed through their fusion with target-specific phage peptides. These phage-displayed peptides can be embedded into liposomes and used to produce tumor-specific liposomes and drug-loading nanocarriers. ${ }^{73}$ Novel peptide ligands targeting high-risk neuroblastoma cells were isolated and characterized using a combination of in vitro and ex vitro PD screening against both neuroblastoma and tumoral cells obtained from murine models. Furthermore, combining the tumor-specific peptides with doxorubicinloaded liposomes caused a meaningful suppression in tumor volume and a promoted survival in neuroblastoma models in a preclinical study. ${ }^{74}$ 
Table I. PD-Derived Therapeutic Drugs That Are Already Approved or Under Clinical Trial. ${ }^{a}$

\begin{tabular}{llll}
\hline Product & Manufacturer & Diseases & Phase \\
\hline Romiplostim (Nplate) & Amgen & Immune thrombocytopenia purpura & Approved \\
Ecallantide (Kalbitor) & Dyax & Acute attacks of hereditary angioedema & Approved \\
Peginesatide (OMONTYS) & Affymax, Takeda & Chronic kidney disease associated anemia & Approved \\
KalbitorW (Ecallantide) & Dyax & Hereditary angioedema (HAE) & Approved \\
AMG-386 & Amgen & Antiangiogenic; killing tumor cells and blocking blood vessels \\
HematideW (Peginesatide) & Affymax, Takeda & Anemia & Chronic kidney disease \\
& & Chronic renal failure & III \\
DX-890 & Dyax/Debiopharm & Cystic fibrosis, chronic obstructive pulmonary disease \\
CNTO530/CNTO 528 & Ortho Biotech & Chronic kidney disease associated anemia \\
AMG 8I9 & Amgen & A peptibody targeting nerve growth factor for pain \\
\hline
\end{tabular}

Abbreviation: PD, phage display.

${ }^{a}$ All of the data summarized in this table were retrieved from Hamzeh-Mivehroud et al ${ }^{3}$ and Omidfar et al. ${ }^{77}$

The in vivo PD approach, which was developed by Ruoslahti in 1996, has been a valuable tool used to isolate organand tissue-specific peptides that bind to cell markers or cell-permeating peptides. The use of in vivo PD allowed for the identification of the synthetic peptide ACSSSPSKHCG. This peptide enables efficacious transdermal drug delivery across skin thanks to the creation of transient opening in the skin barrier, allowing peptide drugs to be delivered to systemic circulation. The coadministration of insulin and this peptide on abdominal skin in a murine model resulted in increased systemic amounts of insulin and reduced glucose concentrations in blood. ${ }^{75}$

\section{Phage Display as Biotechnological Approach for the Development of Therapeutic Drugs}

In 2013, the market for protein-based drugs is over an estimated US $\$ 40$ billion/year. ${ }^{76}$ Therefore, PD libraries may be important reservoirs of clinically promising peptides and peptidomimetics. Table 1 summarizes peptide-based therapeutic drugs that have been produced using PD technology and approved for commercialization or that are in specific phases of clinical trials.

\section{Conclusion}

PD technology involves the expression of sequences of interest inserted together within a gene encoding a viral capsid protein, and a modified target peptide is subsequently displayed on the viral capsid of the phage. Through random nucleotide insertions, the resulting viral particles form a PD peptide library. Since its invention, PD technology has undergone tremendous development with respect to its use in a wide range of promising biomedical applications in different fields, such as oncology, immunology, cell biology, pharmacology, and drug discovery and delivery, and its use in other fields remains to be explored. Therefore, PD is an essential method used to solve traditional pharmacologic problems through the discovery of novel potential drugs or the development of efficient and efficacious drug delivery systems. Thus, because of its great potential, future research should be directed toward combining approaches with relatively novel technologies.

\section{Acknowledgments}

The authors would like to thank Yachay Tech University for the support provided in our projects and ideas.

\section{Author Contributions}

All authors performed the study and approved the final manuscript.

\section{Declaration of Conflicting Interests}

No potential conflicts were declared.

\section{Funding}

No financial support of the research, authorship, and/or publication of this article was declared.

\section{ORCID iD}

Nelson Santiago Vispo (1) https://orcid.org/0000-0002-4081-3984

\section{References}

1. Azzazy HME, Highsmith WE. Phage display technology: clinical applications and recent innovations. Clin Biochem. 2002;35: 425-445.

2. Pande J, Szewczyk MM, Grover AK. Phage display: Concept, innovations, applications and future. Biotechnol Adv. 2010;28: 849-858.

3. Hamzeh-Mivehroud M, Alizadeh AA, Morris MB, et al. Phage display as a technology delivering on the promise of peptide drug discovery. Drug Discov. Today 2013;18:1144-1157.

4. Azzazy HM, Highsmith WE. Phage display technology: clinical applications and recent innovations. Clin Biochem. 2002;35: 425-445.

5. ÁLvarez-Vallina L, Blanco B, Díaz-Espada F, et al. Anticuerpos Monoclonales: Realidades Y Perspectivas. Madrid: Editorial Complutense, https://www.researchgate.net/publication/ 
276270611_Anticuerpos_monoclonales_realidades_y_perspecti vas. Published 2004. Accessed November 16, 2018.

6. Smith GP, Petrenko VA. Phage display. Chem Rev. 1997;97: 391-410.

7. Pande J, Szewczyk MM, Grover AK. Phage display: concept, innovations, applications and future. Biotechnol Adv. 2010;28: 849-858.

8. Arap MA. Phage display technology: applications and innovations. Genet Mol Biol. 2005;28:1-9.

9. Wu C-H, Liu I-J, Lu R-M, et al. Advancement and applications of peptide phage display technology in biomedical science. J Biomed Sci. 2016;23:8.

10. WHO | World Health Organization. http://www.who.int. Accessed May 3, 2018.

11. Craig R, Li S. Function and molecular mechanism of tumortargeted peptides for delivering therapeutic genes and chemical drugs. Mini Rev Med Chem. 2006;6:757-764.

12. Bray F, Ferlay J, Soerjomataram I, et al. Global cancer statistics 2018: GLOBOCAN estimates of incidence and mortality worldwide for 36 cancers in 185 countries. CA Cancer J Clin. 2018;68: 394-424.

13. Dai X, Cai C, Xiao F, et al. Identification of a novel aFGF-binding peptide with anti-tumor effect on breast cancer from phage display library. Biochem Biophys Res Commun. 2014;445:795-801.

14. Park H-Y, Lee K-J, Lee S-J, et al. Screening of peptides bound to breast cancer stem cell specific surface marker CD44 by phage display. Mol Biotechnol. 2012;51(3):212-220.

15. Sun J, Zhang C, Liu G, et al. A novel mouse CD133 bindingpeptide screened by phage display inhibits cancer cell motility in vitro. Clin Exp Metastasis. 2012;29(3):185-196.

16. Fagbohun OA, Bedi D, Jayanna PK, et al. Landscape phage probes for breast cancer cells. In: Technical Proceedings of the 2008 Nanotechnology Conference and Trade Show, NSTI Nanotech. 2008:1-5.

17. Liu F, Qi C-L, Kong M, et al. Screening specific polypeptides of breast cancer stem cells from a phage display random peptide library. Oncol Lett. 2016;12:4727-4731.

18. Phage selection of bicyclic peptides binding Her2. ScienceDirect. https://www.sciencedirect.com/science/article/pii/S004040 2014008126. Accessed November 21, 2018.

19. Estadísticas del cáncer. National Cancer Institute. https:// www.cancer.gov/espanol/cancer/naturaleza/estadisticas. Accessed May 3, 2018.

20. Yeh C-Y, Hsiao J-K, Wang Y-P, et al. Peptide-conjugated nanoparticles for targeted imaging and therapy of prostate cancer. Biomaterials. 2016;99:1-15.

21. Jayanna PK, Bedi D, Deinnocentes P, et al. Landscape phage ligands for PC3 prostate carcinoma cells. Protein Eng Des Sel. 2010;23(6):423-430.

22. Peng Y, Zhang X, Prater A, et al. Targeting aggressive prostate cancer cell-associated CD44v6 using phage display selected peptides. J Nucl Med. 2017;58:624.

23. Wang W, Chen X, Li T, et al. Screening a phage display library for a novel FGF8b-binding peptide with anti-tumor effect on prostate cancer. Exp Cell Res. 2013;319(8):1156-1164.
24. Soendergaard M, Newton-Northup JR, Deutscher SL. Original article in vivo phage display selection of an ovarian cancer targeting peptide for SPECT/CT imaging. Am J Nucl Med Mol Imaging. 2014;4:561-570.

25. Ma C, Yin G, Yan D, et al. A novel peptide specifically targeting ovarian cancer identified by in vivo phage display. J Pept Sci. 2013;19(12):730-736.

26. Wang L, Wang $\mathrm{L}, \mathrm{Hu}$ Y, et al. Identification of a peptide specifically targeting ovarian cancer by the screening of a phage display peptide library. Oncol Lett. 2016;11:4022-4026.

27. Zhou C, Kang J, Wang X, et al. Phage display screening identifies a novel peptide to suppress ovarian cancer cells in vitro and in vivo in mouse models. BMC Cancer. 2015;15:889.

28. Wang C-H, Weng C-H, Che Y-J, et al. Cancer cell-specific oligopeptides selected by an integrated microfluidic system from a phage display library for ovarian cancer diagnosis. Theranostics. 2015;5:431-442.

29. Li HM, Guo K, Yu Z, et al. Diagnostic value of protein chips constructed by lung-cancer-associated markers selected by the T7 phage display library. Thorac Cancer. 2015;6(4):469-474.

30. Lee KJ, Lee JH, Chung HK, et al. Novel peptides functionally targeting in vivo human lung cancer discovered by in vivo peptide displayed phage screening. Amino Acids. 2015;47(2): 281-289.

31. Lee KJ, Lee JH, Chung HK, et al. Application of peptide displaying phage as a novel diagnostic probe for human lung adenocarcinoma. Amino Acids. 2016;48(4):1079-1086.

32. Lee S-M, Lee E-J, Hong H-Y, et al. Targeting bladder tumor cells in vivo and in the urine with a peptide identified by phage display. Mol Cancer Res 2007;5:11-19.

33. Sun X, Niu G, Yan Y, et al. Phage display derived peptides for osteosarcoma imaging. Clin Cancer Res. 2010;16(6):4268-4277.

34. Samoylova TI, Petrenko VA, Morrison NE, et al. Phage probes for malignant glial cells. Mol Cancer Ther. 2003;2(11):1129-1137.

35. Zhang Z, Xu L, Wang Z. Screening serum biomarkers for early primary hepatocellular carcinoma using a phage display technique. J Clin Lab Anal. 2011;25(6):402-408.

36. Zhang W-J, Sui Y-X, Budha A, et al. Affinity peptide developed by phage display selection for targeting gastric cancer. World $J$ Gastroenterol. 2012;18:2053-2060.

37. Bedi D, Gillespie JW, Petrenko VA. Selection of pancreatic cancer cell-binding landscape phages and their use in development of anticancer nanomedicines. Protein Eng Des Sel. 2014;27: 235-243.

38. Dye C. After 2015: infectious diseases in a new era of health and development. Philos Trans R Soc Lond B Biol Sci. 2014;369: 20130426.

39. Portes L da S, Kioshima ES, de Camargo ZP, et al. Subtractive phage display selection for screening and identification of peptide sequences with potential use in serodiagnosis of paracoccidioidomycosis caused by Paracoccidioides brasiliensis. Lett Appl Microbiol. 2017;65(5):346-353.

40. Alban SM, De Moura JF, Thomaz-Soccol V, et al. Phage display and synthetic peptides as promising biotechnological tools for the serological diagnosis of leprosy. PLoS One. 2014;9: e106222. 
41. Link JS, Alban SM, Soccol CR, et al. Synthetic peptides as potential antigens for cutaneous leishmaniosis diagnosis. J Immunol Res. 2017;2017:5871043.

42. Toledo-Machado CM, Bueno LL, Menezes-Souza D, et al. Use of phage display technology in development of canine visceral leishmaniasis vaccine using synthetic peptide trapped in sphingomyelin/cholesterol liposomes. Parasit Vectors. 2015;8:133.

43. WHO | 10 facts on malaria. WHO, http://www.who.int/features/ factfiles/malaria/en/. Accessed May 3, 2018.

44. Eda K, Eda S, Sherman IW. Identification of peptides targeting the surface of Plasmodium falciparum-infected erythrocytes using a phage display peptide library. Am J Trop Med Hyg. 2004;71(2):190-195.

45. Cheong FW, Fong MY, Lau YL. Identification and characterization of epitopes on Plasmodium knowlesi merozoite surface protein-142 (MSP-142) using synthetic peptide library and phage display library. Acta Trop. 2016;154:89-94.

46. Baldwin MR, Li X, Hanada T, et al. Merozoite surface protein 1 recognition of host glycophorin A mediates malaria parasite invasion of red blood cells. Blood. 2015;125:2704-2711.

47. Vega-Rodriguez J, Perez-Barreto D, Ruiz-Reyes A, et al. Targeting molecular interactions essential for Plasmodium sexual reproduction. Cell Microbiol. 2015;17(11):1594-1604.

48. Ghosh a K, Ribolla PE, Jacobs-Lorena M. Targeting Plasmodium ligands on mosquito salivary glands and midgut with a phage display peptide library. Proc Natl Acad Sci U S A. 2001;98(23): 13278-13281.

49. Mukherjee D, Mishra P, Joshi M, et al. EWGWS insert in Plasmodium falciparum ookinete surface enolase is involved in binding of PWWP containing peptides: Implications to mosquito midgut invasion by the parasite. Insect Biochem Mol Biol. 2016;68:13-22.

50. Ruiz A, Pérez D, Muñoz MC, et al. Targeting essential Eimeria ninakohlyakimovae sporozoite ligands for caprine host endothelial cell invasion with a phage display peptide library. Parasitol Res. 2015;114(11):4327-4331.

51. Yang L, Cen J, Xue Q, et al. Identification of rabies virus mimotopes screened from a phage display peptide library with purified dog anti-rabies virus serum IgG. Virus Res. 2013; 174(1-2):47-51.

52. Larralde OG, Martinez R, Camacho F, et al. Identification of hepatitis A virus mimotopes by phage display, antigenicity and immunogenicity. J Virol Methods. 2007;140(1-2):49-58.

53. Lam C-W, AbuBakar S, Chang L-Y. Identification of the cell binding domain in Nipah virus $\mathrm{G}$ glycoprotein using a phage display system. J Virol Methods. 2017;243:1-9.

54. Liu K, Huan B, Shao D, et al. Peptide P3 selected from phage display screen shows antiviral activity against porcine reproductive and respiratory syndrome virus [published online ahead of print February 24, 2016]. J Biomed Sci. doi:10.4172/2254-609X. 100024.

55. Yin L, Luo Y, Liang B, et al. Specific ligands for classical swine fever virus screened from landscape phage display library. Antiviral Res. 2014;109:68-71.
56. Wang M, Li X, Chen J, et al. Screening and evaluating the mimic peptides as a useful serum biomarker of ankylosing spondylitis using a phage display technique. Rheumatol Int. 2011;31(8): 1009-1016.

57. Weng X, Liao Q, Li K, et al. Screening serum biomarker of knee osteoarthritis using a phage display technique. Clin Biochem. 2012;45(4-5):303-308.

58. Araujo GR, Vaz ER, Fujimura PT, et al. Improved serological detection of rheumatoid arthritis: a highly antigenic mimotope of carbonic anhydrase III selected in a murine model by phage display. Arthritis Res Ther. 2015;17:168.

59. Sun Y, Tan J, Yin X, et al. Regulation of osteoblast differentiation by affinity peptides of TGF- $\beta 1$ identified via phage display technology. ACS Biomater Sci Eng. 2018;4(7):2552-2562.

60. Park JP, Cropek DM, Banta S. High affinity peptides for the recognition of the heart disease biomarker troponin I identified using phage display. Biotechnol Bioeng. 2010;105(4):678-686.

61. Yang J, Fuller PJ, Morgan J, et al. Use of phage display to identify novel mineralocorticoid receptor-interacting proteins. Mol Endocrinol. 2014;28(9):1571-1584.

62. Padmanaban G, Park H, Choi JS, et al. Identification of peptides that selectively bind to myoglobin by biopanning of phage displayed-peptide library. J Biotechnol. 2014;187:43-50.

63. Ghoshal S, Bondada V, Saatman KE, et al. Phage display for identification of serum biomarkers of traumatic brain injury. J Neurosci Methods. 2016;272:33-37.

64. Li J, Zhang Q, Pang Z, et al. Identification of peptide sequences that target to the brain using in vivo phage display. Amino Acids. 2012;42:2373-2381.

65. Linzhi Y, Dan C, Arhin SK, et al. Screening for novel peptides specifically binding to the surface of ectopic endometrium cells by phage display. Cell Mol Biol (Noisy-le-grand). 2018;64:36-40.

66. Zhao Y, Wang Q, Jin Y, et al. Discovery and characterization of a high-affinity small peptide ligand, H1, targeting FGFR2IIIc for skin wound healing. Cell Physiol Biochem. 2018;49(3): 1033-1048.

67. Suire CN, Nainar S, Fazio M, et al. Peptidic inhibitors of insulindegrading enzyme with potential for dermatological applications discovered via phage display. PLoS One. 2018;13:e0193101.

68. Sakamoto K, Kanematsu-Yamaki Y, Kamada Y, et al. Identification of ligand-selective peptidic ActRIIB-antagonists using phage display technology. Biochem Biophys Rep. 2017;11:33-39.

69. Westwater C, Kasman LM, Schofield DA, et al. Use of genetically engineered phage to deliver antimicrobial agents to bacteria: an alternative therapy for treatment of bacterial infections. Antimicrob Agents Chemother. 2003;47:1301-1307.

70. Vaks L, Benhar I. In vivo characteristics of targeted drug-carrying filamentous bacteriophage nanomedicines. J Nanobiotechnol. 2011;9:58.

71. Yacoby I, Bar H, Benhar I. Targeted drug-carrying bacteriophages as antibacterial nanomedicines. Antimicrob Agents Chemother. 2007;51:2156-2163.

72. Harhala M, Barylski J, Humińska-Lisowska K, et al. Two novel temperate bacteriophages infecting Streptococcus pyogenes: their 
genomes, morphology and stability. PLoS One. 2018;13: $\mathrm{e} 0205995$.

73. Jayanna PK, Torchilin VP, Petrenko VA. Liposomes targeted by fusion phage proteins. Nanomedicine. 2009;5(1):83-89.

74. Loi M, Di Paolo D, Soster M, et al. Novel phage display-derived neuroblastoma-targeting peptides potentiate the effect of drug nanocarriers in preclinical settings. J Control Release. 2013; 170(2):233-241.
75. Chen Y, Shen Y, Guo X, et al. Transdermal protein delivery by a coadministered peptide identified via phage display. Nat Biotechnol. 2006;24:455-460.

76. Bruno BJ, Miller GD, Lim CS. Basics and recent advances in peptide and protein drug delivery. Ther Deliv. 2013;4:1443-1467.

77. Omidfar K, Daneshpour M. Advances in phage display technology for drug discovery. Expert Opin Drug Discov. 2015;10(6): 651-669. 